\title{
The Blood Levels of the Amino Acid Kynurenine and Indoloxi- genase are Predictive of Acute Graft Versus Host Disease (aGVHD)
}

\author{
Ferreira DC*, Silva IDCG, Lo Turco EG and Oliveira JSR \\ Department of Hematology and Hemotherapy, Federal University of São Paulo, Brazil
}

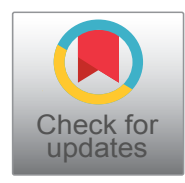

*Corresponding author: Ferreira DC, Department of Hematology and Hemotherapy, Federal University of São Paulo, Brazil

\begin{abstract}
The main barrier to the success of hematopoietic stem cell transplantation (HSCT) is acute graft versus host disease (aGVHD). The risk factors for aGVHD are mainly clinical and can be improved if additional biological factors are incorporated, such as the metabolomic profile. It is known that the levels of various metabolites measured are important in the process of immunoregulation [1]. In our population study we investigated the systemic metabolic profile for 26 (Group I) allotransplant recipients. The metabolomics study was carried out by Targeted Mass Spectrometry. Plasma samples of the 26 patients were collected before the Conditioning Regimen of HSCT. All patients were clinically evaluated until 2 years after transplant and then Group I was subdivided into subgroup IA: 16 patients that presented aGVHD and subgroup IB: 10 patients without aGVHD. When comparing the metabolites dosages from subgroup IA with those of subgroup IB, it was observed that 25 metabolites We then verified by bioinformatics analyses that the ratio Kynurenine/Tryptophan which estimates the Indoloxigenase (IDO) enzyme activity. Kynurenine and the IDO enzyme are metabolites that present clinical evidence in the literature regarding the immunotolerance process. The role of IDO enzyme in the maintenance of the allogeneic fetus in pregnant women was verified in an animal model in 1998 by Mellor and Munn. Thus, the pre-transplant analysis of the metabolomics profile of allotransplant recipients seems to contribute to a better understanding of immunological process and lower dosages of Kynurenine and IDO activity enzyme pre-transplant can identify patients at higher risk for developing aGVHD.
\end{abstract}

\section{Introduction}

Allogeneic hematopoietic stem cell transplantation (HSCT) represents an effective therapeutic strat- egy for a variety of onco-hematologic conditions. The improvement of the technique and the development of new strategies have expanded its use, however the complications observed in patients submitted to HSCT remain a limiting factor in survival [1]. The indication for HSCT should be individualized and based on the expectated long-term disease-free survival with conventional chemotherapy versus the risk of relapse and the risk of treatment related mortality/morbidity after transplantation. Some strategies based on pre-transplantation prognostic factors are associated with long term survival [2]. However, these factors are mainly clinical and can be improved if additional biological factors are incorporated, for example the pre-transplant metabolomic profile that differs between allotransplantation recipients with and without post-transplant acute Graft-versus-host Disease (aGVHD) [3].

Currently, available diagnostic and staging tools are not able to identify patients with an increased risk of morbidity and mortality due to aGVHD. In parallel, the past few years have been characterized by explosive evolution of genomics technologies, largely due to technological advances in chemistry, engineering and bioinformatics. Based on these opportunities, plasma biomarkers have been identified and validated as promising diagnostic and prognostic tools for aGVHD [4].

The metabolomics presents its greatest impact in research in the field of nutrition, medicine and the

Citation: Ferreira DC, Silva IDCG, Lo Turco EG, Oliveira JSR (2020) The Blood Levels of the Amino Acid Kynurenine and Indoloxigenase are Predictive of Acute Graft Versus Host Disease (aGVHD). Int J Stem Cell Res Ther 7:065. doi.org/10.23937/2469-570X/1410065

Accepted: January 23, 2020: Published: January 25, 2020

Copyright: (c) 2020 Ferreira DC, et al. This is an open-access article distributed under the terms of the Creative Commons Attribution License, which permits unrestricted use, distribution, and reproduction in any medium, provided the original author and source are credited. 
Table 1: Description of the clinical characteristics of Group I: 26 patients submitted to HSCT.

\begin{tabular}{|c|c|}
\hline Clinical Parameters & $N=26(100 \%)$ \\
\hline \multicolumn{2}{|l|}{ Gender } \\
\hline Male & $16(61 \%)$ \\
\hline Female & $10(39 \%)$ \\
\hline \multicolumn{2}{|l|}{ Age (years) Median: 36 (range 18-65) } \\
\hline 18 a 50 & $19(73 \%)$ \\
\hline$\geq 50$ & $7(27 \%)$ \\
\hline \multicolumn{2}{|l|}{ Diagnosis } \\
\hline AML, ALL, SMD & $15(61 \%)$ \\
\hline LH, LNH & $7(27 \%)$ \\
\hline CML & $1(3.8 \%)$ \\
\hline MM & $1(3.8 \%)$ \\
\hline AA & $2(7.6 \%)$ \\
\hline \multicolumn{2}{|l|}{ Donor Type } \\
\hline Related & $20(76.9 \%)$ \\
\hline Non Related & $6(23.1 \%)$ \\
\hline \multicolumn{2}{|l|}{ HLA Match } \\
\hline $10 / 10$ & $23(88.5 \%)$ \\
\hline 09/10 (misA) & $1(3.8 \%)$ \\
\hline Haplo & $2(7.7 \%)$ \\
\hline \multicolumn{2}{|l|}{ Conditioning Regimen } \\
\hline Myeloablative & $16(61.5 \%)$ \\
\hline Reduced Intensity Conditioning & $10(38.5 \%)$ \\
\hline \multicolumn{2}{|l|}{ Prophilaxis of GVHD } \\
\hline $\mathrm{CSA}+\mathrm{MTX}$ & $22(85.6 \%)$ \\
\hline $\mathrm{CSA}+\mathrm{MMF}$ & $4(15.4 \%)$ \\
\hline \multicolumn{2}{|l|}{ Disease status pre transplant } \\
\hline $1^{\text {a }} \mathrm{CR}$ (First Complete Remission) & $9(34.6 \%)$ \\
\hline $2^{a}$ CR (Second Complete Remission) & $2(7.7 \%)$ \\
\hline $3^{\text {a }}$ CR (Third Complete Remission) & $3(11.5 \%)$ \\
\hline No remission & $10(38.5 \%)$ \\
\hline Bone Marrow Aplasia & $2(7.7 \%)$ \\
\hline \multicolumn{2}{|l|}{ Source of Stem Cell } \\
\hline Peripheral blood (PB) & $17(65 \%)$ \\
\hline Bone Morrow (BM) & $9(35 \%)$ \\
\hline
\end{tabular}

AML: Acute Myelogenous Leukemia; ALL: Acute Lymphoblastic Leukemia; MS: Myelodysplastic Syndromes; HL: Hodgkin's Lymphoma; NHL: Non-Hodgkin's Lymphoma; CML: Chronic Myelogeneous Leukemia; MM: Multiple Myeloma; AA: Aplastic Anemia; Bu: Busulphan; Cy: Cyclophosphamida; TBI: Total Body Irradiation; ATG: Anti-Thymoglobulin; Flu: Fludarabina; Mel: Melphalan; CSA: Cyclosporin; MTX: Methotrexate; SM: Sodium Mycophelonate; aGVHD: Acute Graft Versus Host Disease.

pharmaceutical industry through the discovery of key biomarkers which allow the early detection of the onset of the disease, or ideally, identify a condition before the appearance of the disease, the pre-disease state. This technique offers innumerable possibilities in the study of pathophysiological conditions and will allow the prevention of diseases, as well as
Table 2: Description and characteristics of Acute Graft Versus Host Disease.

\begin{tabular}{|l|l|l|l|}
\hline Ocurrence & N & Median & Range \\
\hline With aGVHD & $16(61.5 \%)$ & $\mathrm{D}+55$ & $(23-119)$ \\
\hline Without aGVHD & $10(38.5 \%)$ & - & - \\
\hline Characteristics & $\mathrm{N}=16(\%)$ & & \\
\hline Staging of aGVHD & & & \\
\hline I/II & $9(56.25 \%)$ & & \\
\hline III & $3(18.75 \%)$ & \\
\hline IV & $4(25 \%)$ & \\
\hline Organ Affected by aGVHD & & \\
\hline Skin & $6(37.5 \%)$ & \\
\hline Gastrointestinal Tract & $2(12.5 \%)$ & \\
\hline Liver & $0(0.0 \%)$ & \\
\hline 2 organs affected & $5(31.25 \%)$ & \\
\hline 3 organs affected & $3(18.75 \%)$ & \\
\hline
\end{tabular}

aGVHD: acute Graft Versus Host Disease.

the provision of adequate clinical treatment through specific medications. In addition, the metabolomic phenotype will provide an enormous opportunity to evaluate the metabolic response in the treatment of each patient, making individualized clinical treatment possible [4].

In this context, we have investigated whether metabolites identified prior to stem cell transplantation may reflect a risk of developing aGVHD in the post-transplant period.

\section{Materials and Methods}

The study was approved by the Research Ethics Committee of the hospitals São Paulo and Santa Marcelina and samples were collected after written informed consent. The study included 26 consecutively allotransplanted adult patients ( 16 men and 10 women, median age 36 with range 18-65 years) in which clinical evaluation and serial analysis of plasma metabolites were performed. The decision to perform an allotransplant was taken by the Bone Marrow Transplant Services of São Paulo and Santa Marcelina Hospitals and based on national guidelines. Thus, our study is population-based and includes an unselected and consecutive group of well characterized patients.

Clinical follow-up of the patients included in the study from pre-transplant period ( $D-8)$ to $D+2$ years was performed. The clinical data were collected through evaluations and records made in medical records. The data collected were: Diagnosis, type of transplantation, age, gender, source of cells used for transplantation, patient and donor HLA, conditioning regimen, GVHD-prophylaxis regimen, day of aGVHD , organ affected by aGVHD, GVHD-staging. The clinical characteristics and occurrence of aGVHD of these patients are given in Table 1 and Table 2, respectively. 


\section{Sample preparation, groups, analysis of metabo- lite and bioinformatical analyses}

Blood samples were collected before HSCT from 26 patients $(\mathrm{Gl})$. During the post-transplant follow up patients were then subdivided into two subgroups between those who developed (GIA) or did not develop (GIB) the acute Graft-Versus-Host Disease. A group of 166 healthy persons from the same neighborhood of São Paulo city was taken from a biobank to constitute the control group.

All venous blood samples were collected into sterile plastic tubes (BD Vacutainer $R$, tubos de separação de soro SSTTM, Becton-Dickenson; Franklin Lakes, NJ, EUA). After collection of whole blood in tube with anticoagulant (EDTA), the samples were centrifuged for 10 minutes. The blood plasma of each sample was separated into two $1.5 \mathrm{cc}$ microtubes and frozen at $-80^{\circ} \mathrm{C}$ until analyzed.

Quantitative analysis of the metabolites was performed using Targeted Quantitative MS/MS using the AbsolutIDQ kit p180 (Biocrates Life Science, Innsbruck, Austria). Briefly, a targeted profiling scheme is used to quantitatively screen small molecules of known metabolites, using multiple reaction monitoring, neutral loss, and precursor ion scans. Quantitation of the metabolites of the biological sample is done by reference to appropriate internal standards. The concentrations of all variables are reported in microMolar and the metabolite panel is composed of 186 different metabolites, of which 40 acetylcarnitines, 19 amino acids, ornithine and citrulline, 19 biogenic amines, sum of Hexoses, 76 phosphatidylcholines, 14 phosphatidylcholines and 15 sphingomyelins (Annex 1).
The dosage of the metabolites performed in duplicate was evaluated for the discovery of possible biomarkers that could be associated with any clinical variable (aGVHD) through logistic regression analysis. Quantification of metabolite concentration and quality control evaluation were automatically calculated on microMo$\operatorname{lar}(\mu \mathrm{M})$ MetIQ software (BIOCRATES Life Sciences AG, Innsbruck, Austria). Metabolites below detection limits determined by the program itself were excluded from subsequent analyzes. An excel file was exported containing the names and concentrations of the metabolites analyzed with the concentration in $\mathrm{mmol} / \mathrm{L}$ of plasma. The data was sent to Metabo Analyst 3.0 software (www.metaboanalyst.ca), and transformed into Log2 in the software itself.

For statistical analysis, the Pearson correlation coefficient was used, considering the significance value of less than $5 \%(p<0.05)$, analysis of variance (ANOVA) and characteristic curves of receiver operation (ROC) were constructed from the most important variables created by the discriminatory least squares analysis model (PLSDa). A total of 186 metabolites were analysed by Targeted Mass Spectrometry in all samples.

\section{Results}

Table 1, Table 2, Graphic 1 and Table 3.

According to the Pearson's Correlation Test, in the table below, it can be observed the top 25 metabolites of pretransplant samples that positively (Salmon) or negatively (Blue) are correlated to aVGHD.

\section{Graphic 2, Graphic 3, Graphic 4 and Graphic 5.}

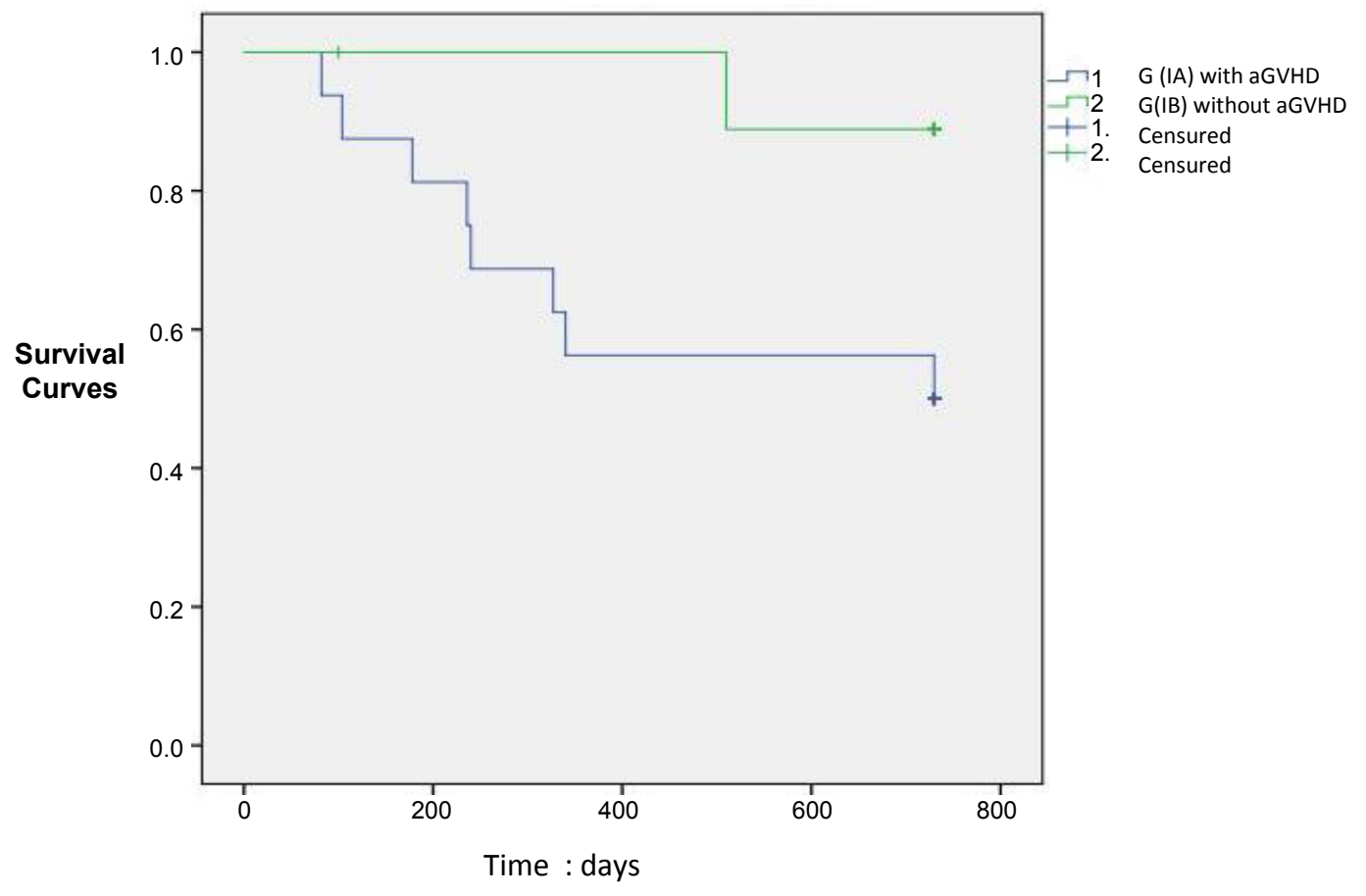

Graphic 1: Survival Curves of patiens with (IA) and without aGVHD (IB) $(p<0.05)$. 
Table 3: Correlation Coefficient analysis of Metabolites with aGVHD.

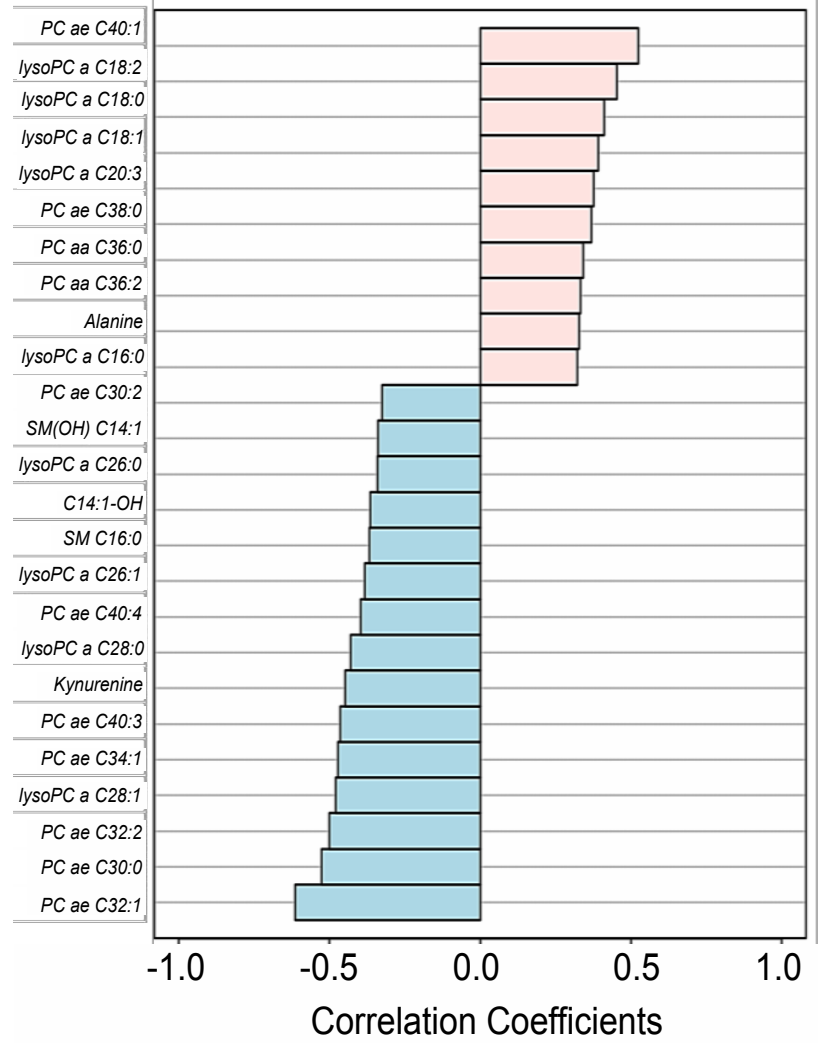

Correlation Coefficients.

\section{Discussion}

In the present study, Group I consisted of 26 patients submitted to allogeneic HSCT. The median age at the time of HSCT was 36 years, ranging from 18 to 65 years. Sixty-one (61\%) were men and $39 \%$ were women. Acute leukemias and myelodysplastic syndromes were the main indications of transplantation, corresponding to $61 \%$ of the patients in the study, and about half $(50 \%)$ of the underlying diseases were in complete remission at the time of HSCT.

The treatment of myelodysplasia and acute leukemia (myeloid and lymphoid) are the main indications of allogeneic transplantation, accounting for about $65 \%$ of this procedure in the Center for International Blood and Marrow Transplant Research (CIBMT) [5].

Improvements in infectious prophylaxis, immunosuppressive drugs, supportive care and HLA-based DNA typing have also contributed to improve outcomes after allogeneic HSCT. However, the main complication of allogeneic HSCT is graft versus host disease (GVHD) which continues to be lethal and limits the use of this important therapy.

\begin{tabular}{|l|l|l|l|l|}
\hline Metabolites & Correlation & T-Stat & P-Value & FDR \\
\hline$P C$ ae C40:1 & 0.52462 & 3.0189 & 0.0059328 & 0.33422 \\
\hline lysoPC a C18:2 & 0.45395 & 2.4959 & 0.019835 & 0.41062 \\
\hline IysoPC a C18:0 & 0.41168 & 2.2131 & 0.036652 & 0.56311 \\
\hline IysoPC a C18:1 & 0.3911 & 2.0818 & 0.048194 & 0.62652 \\
\hline IysoPC a C20:3 & 0.37616 & 1.9888 & 0.058235 & 0.6317 \\
\hline PC ae C38:0 & 0.36909 & 1.9455 & 0.063513 & 0.6317 \\
\hline PC aa C36:0 & 0.3412 & 1.7782 & 0.088036 & 0.71071 \\
\hline PC aa C36:2 & 0.33255 & 1.7275 & 0.096927 & 0.71071 \\
\hline Ala & 0.32852 & 1.704 & 0.1013 & 0.71071 \\
\hline lysoPC a C16:0 & 0.32267 & 1.6701 & 0.1079 & 0.71071 \\
\hline$P C$ ae C30:2 & -0.32447 & -1.6805 & 0.10583 & 0.71071 \\
\hline SM(OH) C14:1 & -0.33938 & -1.7675 & 0.089856 & 0.71071 \\
\hline IysoPC a C26:0 & -0.33943 & -1.7678 & 0.089801 & 0.71071 \\
\hline C14:1-OH & -0.36432 & -1.9165 & 0.067282 & 0.6317 \\
\hline SM C16:0 & -0.36736 & -1.935 & 0.064858 & 0.6317 \\
\hline IysoPC a C26:1 & -0.38312 & -2.0319 & 0.053371 & 0.6317 \\
\hline$P C$ ae C40:4 & -0.39619 & -2.1139 & 0.045105 & 0.62652 \\
\hline IysoPC a C28:0 & -0.42952 & -2.3301 & 0.028534 & 0.48223 \\
\hline Kynurenine & -0.44756 & -2.4519 & 0.021867 & 0.41062 \\
\hline PC ae C40:3 & -0.46366 & -2.5637 & 0.017045 & 0.41062 \\
\hline PC ae C34:1 & -0.47095 & -2.6153 & 0.015168 & 0.41062 \\
\hline IysoPC a C28:1 & -0.47926 & -2.6752 & 0.013239 & 0.41062 \\
\hline$P C$ ae C32:2 & -0.49994 & -2.828 & 0.0093039 & 0.39309 \\
\hline$P C$ ae C30:0 & -0.52521 & -3.0236 & 0.0058671 & 0.33422 \\
\hline$P C$ ae C32:1 & -0.61272 & -3.7982 & $8.76 \mathrm{E}-04$ & 0.14804 \\
\hline
\end{tabular}

Two patients underwent a haplo-identical transplant in this study, being a 27-year-old man and a 62-year-old woman diagnosed with Hodgkin's lymphoma and myelodysplastic syndrome, respectively. Both transplants were non-myeloablative with conditioning regimen including Cyclophosphamide $30 \mathrm{mg} / \mathrm{kg}$, Fludarabine 150 $\mathrm{mg} / \mathrm{m}^{2}$ and Total body irradiation (TBI) 200 c Gy with peripheral blood stem cell source, post-transplant immunosuppression with Cyclophosphamide $50 \mathrm{mg} / \mathrm{kg}$ in $D+3$ and $D+4$, in addition to cyclosporin and mycophenolate from $D+5$. [6].

Of these 26 patients, 16 were diagnosed with aGVHD, which constituted group IA. The other 10 patients who did not develop aGVHD were the IB group. Graphic 1 shows the survival curves of the groups with and without aGVHD, respectively, $50 \%$ and $90 \%$. Therefore, the mortality in Group IA was 50\% (eight patients), while the mortality in Group IB was $10 \%$ (one patient) in the study period and this difference was statistically significant $(p=0.01)$.

A total of 186 Metabolites were analysed by Targeted Mass Spectrometry in all samples. We then evaluated specifically the metabolites that presented 


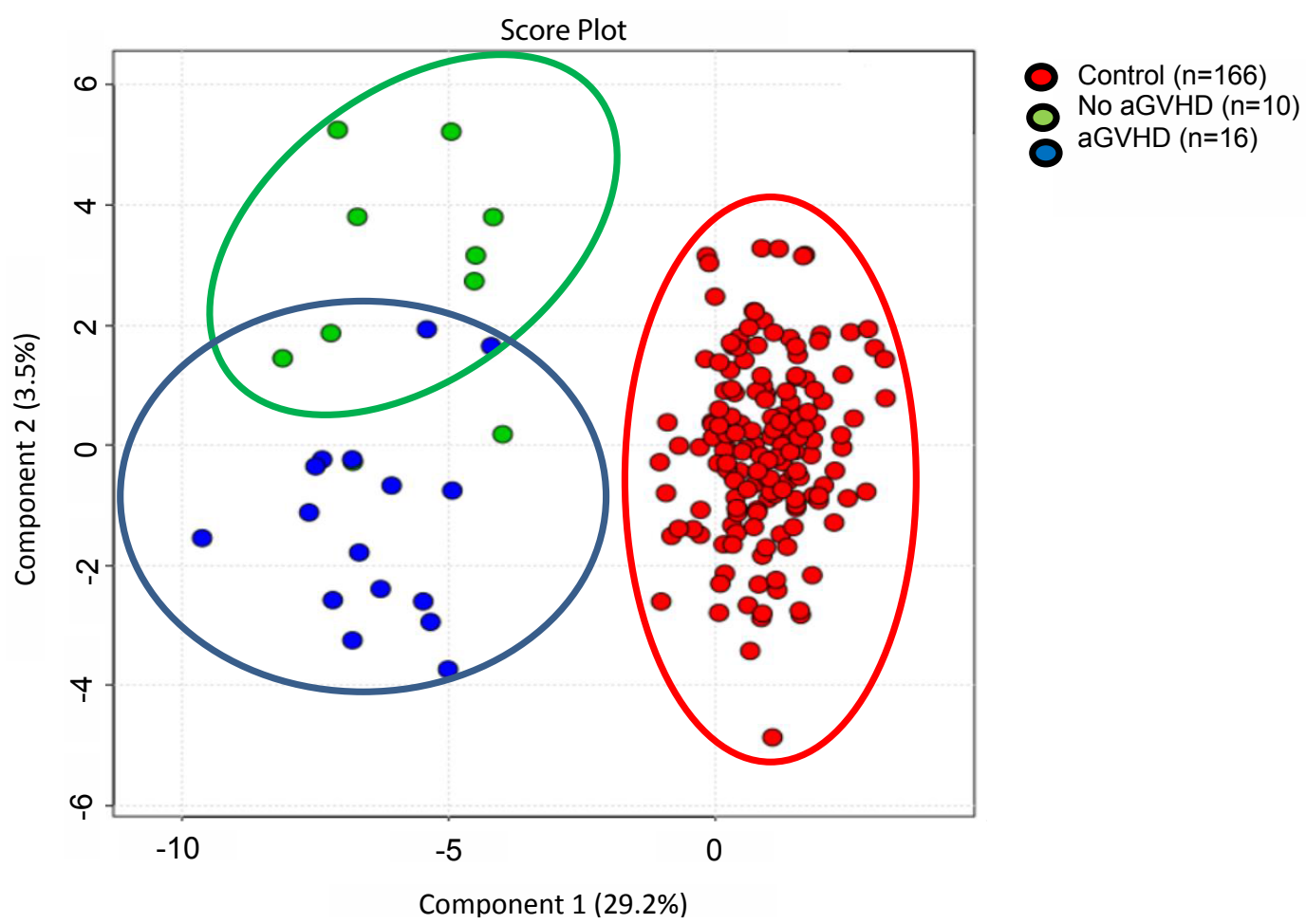

Graphic 2: S-PLSDA analysis demonstrating the existence of metabolic peculiarities in each group that segregate at baseline, not only patients who developed aGVHD from who did not developed aGVHD but also both groups of patients from control group.

Indoloxigenase (Kyn/Trp)
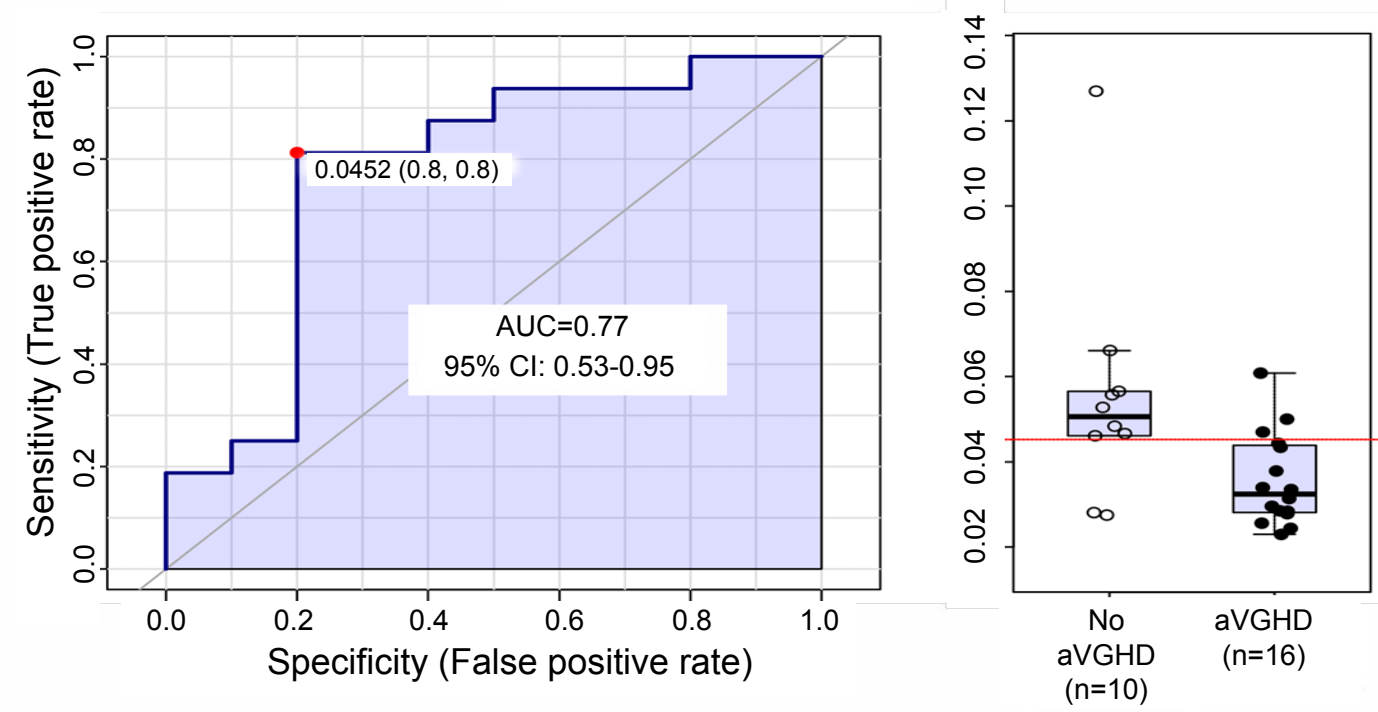

Graphic 3: ROC curve univariate analysis demonstrating the aGVHD predictive performance of indoloxigenase activity, demonstrated by the values generated by the ratio product/substrate of kynurenine/tryptophan (a).

a statistically significant correlation coefficient (Pearson) with aGVHD in pre-transplant samples. Of all identified metabolites found to have significant correlation, Kynurenine called attention because of its intrinsic relationship with the enzyme IDO.

Kynurenine is the primary product of degradation of the essential amino acid tryptophan by the hepatic enzyme Indolamine 2,3 dioxygenase (IDO). IDO is produced on demand, in many tissues in response to immunological signals arriving from white blood cells in situ, leading to acute local depletion of tryptophan and induction of bacteriostasis in parallel inhibition of T-cell activation $(4,5)$ and kynurenine, the product of tryptophan catabolism, regulates T-cell growth and survival (5).

It is also Known that Kynurenine is an endogenous ligand that acts on the aromatic hydrocarbon receptor (ARH), making it a transcription factor that acts by suppressing antitumor immune responses and promoting the survival and motility of tumor cells. Immunologists have identified the role of ARH in T-cell differentiation 


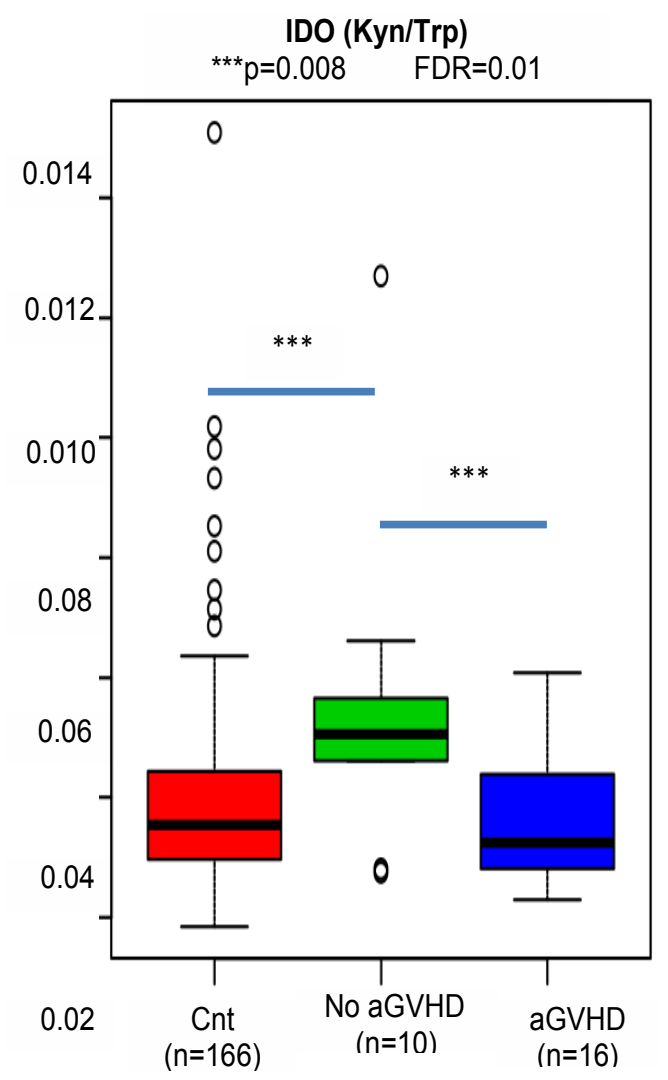

\section{IDO (Kyn/Trp)}

Corr. $=-0.53 p=0.005$ FDR $=0.34$

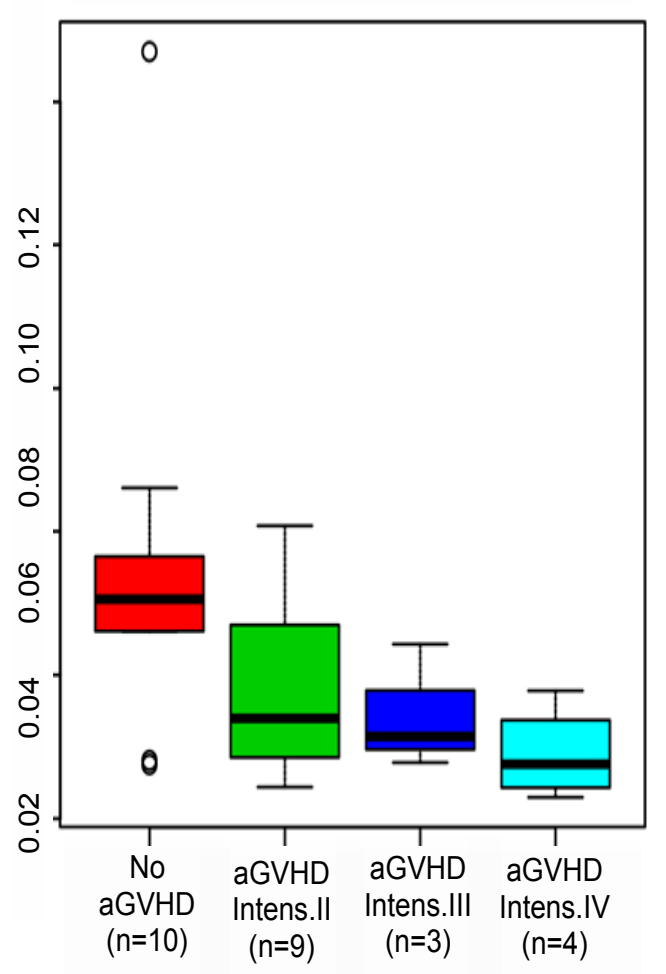

Graphic 4: ANOVA analysis demonstrating the aGVHD associations of ratios having kynurenine as nominator. The reduction in IDO activity is significantly correlated with the aGVHD intensity, the lower the IDO activity the higher the intensity.
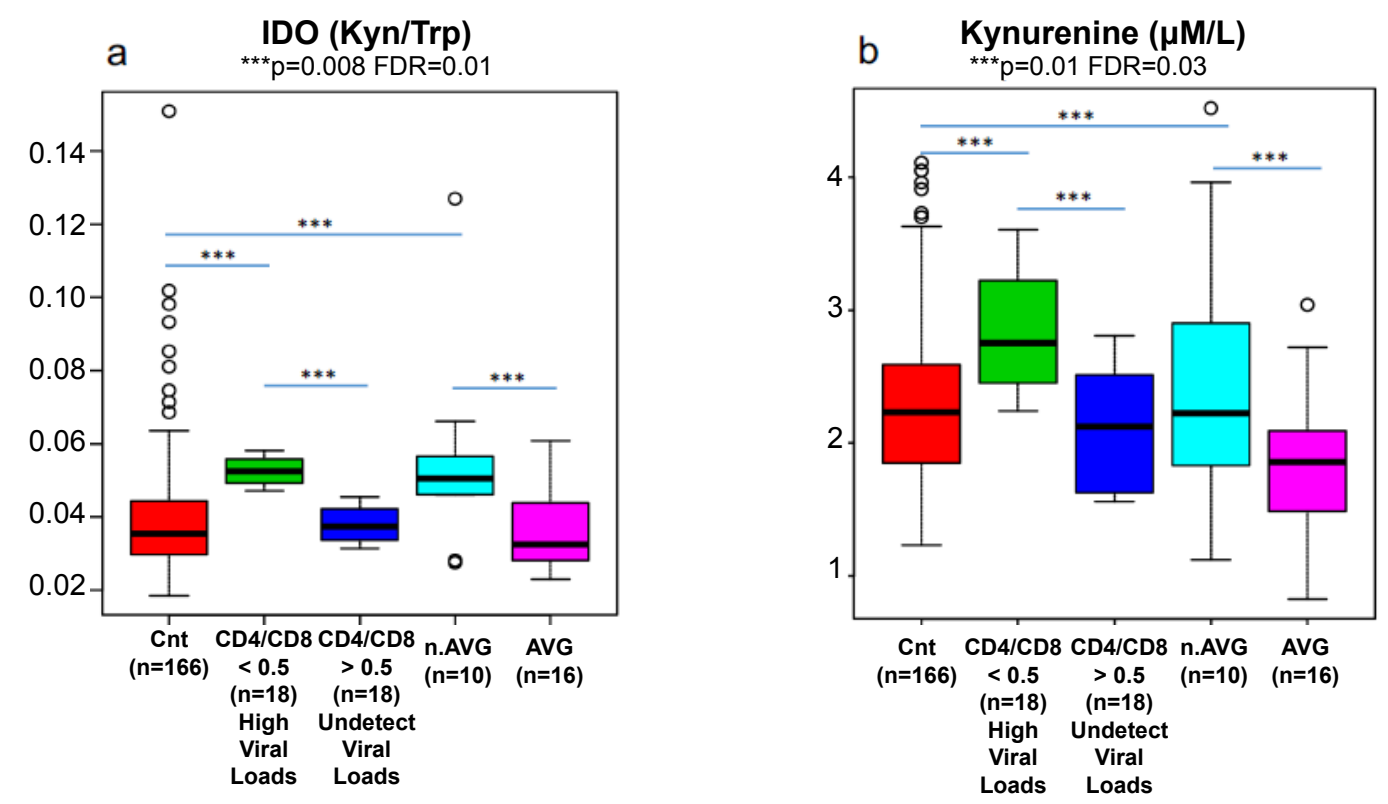

Graphic 5: Ido Activity and Kynurenine dosages of allo-transplanted patients with and without aGVHD compared with 18 HIV patients with High and Undetectable Viral Loads.

as promoter ligands for Treg cell generation and Th17 cell differentiation, both in vivo and in vitro [7]. Kynurenine is produced in sufficient quantities to activate human AHR during cancer progression and inflammation of the local microenvironment, with accumulation of this metabolite resulting in subsequent tolerogenic effects, including increased T-cell regulatory activity by a mechanism dependent on AHR. These results provide evidence of a previously unidentified pathophysiological function of AHR with profound implications for cancer and immunology.

The enzyme indolamine 2,3-dioxygenase (IDO) occupies the main role of connecting the immune system with the kynurenine pathway. There is evidence of the immunosuppressive effects of IDO. Subsequent to the activation of interferon-mediated antigen pre- 
senting cells, the induction of IDO and the kynurenine pathway exert counter-regulatory effects, responsible for the maintenance of homeostasis. Inhibition of $T$ cell function, activation of regulatory $T$ cells and natural inhibition of Natural Killers cells are among the immunosuppressive effects of IDO and Kynurenine [8].

A study of renal transplant patients for more than 5 years, whose inclusion criterion was preserved renal function (Glomerular Filtration Rate $\geq 60 \mathrm{~mL} / \mathrm{min}$ ), identified the presence of Foxp3 expressing Treg cells, as well as expressing Bregs cells of interleukin 10 and dendritic cells expressing the enzyme IDO, as being responsible for immunological tolerance-inducing effects and at the same time counter-regulators of the inflammatory process. Therefore, these cellular subpopulations are most likely to participate in the maintenance of allograft immune quiescence in renal transplant recipients with excellent long-term graft function [9].

It is interesting to note that in 1953 the researcher Medawar considered 3 possible mechanisms to explain the paradox of fetal survival during pregnancy: 1) The anatomical separation between mother and fetus 2) The fetal antigenic immaturity and 3) The mother's immunological tolerance. There is evidence that the repertoire of maternal T cells is transiently affected and tolerates paternal alloantigens (HLA Class I) inherited by the fetus. Therefore, the first two mechanisms cannot explain fetal survival and more attention has been paid to this third mechanism. Certain macrophages induce the production of IDO in response to gamma interferon and inhibit $\mathrm{T}$ cell proliferation in vitro through the rapid consumption of tryptophan [10].

It has been known more than a century that IDO, the catabolizing enzyme of tryptophan, is expressed by the cells of the syncytiotrophoblasts [11] and during normal pregnancy there is a decrease in the systemic concentration of tryptophan [12].

Munn, et al. [10] proposed that IDO-mediated tryptophan depletion is responsible for the maintenance of allogeneic fetuses of pregnant women in an animal model (mice). In 1998, through experiments in mice, these researchers demonstrated that the blockade of the activity of the enzyme IDO through the administration of 1-methyltryptophan resulted in rapid allogenic rejection of the fetus in an animal model, but comparatively when administered in syngeneic fetuses, they did not present rejection. These experiments demonstrated the immunological tolerance exerted by IDO during pregnancy in mice. Thus, these authors reported for the first time that the catabolization of tryptophan by the enzyme IDO leads to an inhibition of T cell proliferation against the developing fetus, which protects itself from eventual rejection.
Other researchers also performed the insertion of the human IDO gene into dendritic cells and after testing the ability to suppress $T$ cells in vitro. As expected, tryptophan concentration decreased while metabolites of the kinurenin pathway increased and $T$ cells were suppressed. These authors suggested that cellular apoptosis is the mechanism of permanent inhibition of these metabolites [13].

In the present study, we could demonstrate in patients submitted to hematopoietic allotransplant, that lower baseline IDO activity and Kynurenine dosages before transplant are significantly associated to future aGVHD. Besides, dividing the patients who did developed aGVHD by its severity, we could also identify an inverse correlation of aGVHD intensity and IDO levels.

To validate, at what extend, our findings were connected to immunological aspects of T cells activity, we analyzed the ratio Kyn/Trp in parallel to CD4/CD8 from the same HIV patients during periods of high and undetectable viral loads.

Results demonstrated a remarkable similarity in terms of elevated IDO activity between patients, at baseline, that did not develop aGVHD and HIV participants experiencing high viral loads.

On the other hand, lower and very similar values, in IDO activity, were detected in the control group, in patients that developed aGVHD and HIV patients during periods of untraceable viral loads.

Important, tryptophan is the unique essential/indispensable amino acid whose metabolism is unable to be reversed to regenerate Trp again. As such, blood levels of this amino acid are highly dependent on ingestion, this possibility, at least theoretically, could explain the immune misbalanced scenarios observed in unappropriated nutritional states. At the same time, it becomes very tempting to speculate about the possibility to up or down-regulate $T$ cells activity just by introducing or removing specific essential/indispensable amino acids.

Although this is a small case study, its results have been relevant and new questions have arisen such as: What would be the role of the Kynurenine and IDO enzyme pathway in aGVHD? How much could metabolomic evaluation could increase the possibilities of better risk stratification, diagnosis and treatment in aGVHD? [14-20].

\section{Conclusion}

Metabolomic analysis through Targeted Mass Spectrometry can be used as an instrument to identify biomarkers and these may reflect the risk of developing acute GVHD. Our research suggest the pre-transplant analysis of the metabolomics profile of allotransplant recipients seems to contribute to a better understanding of immunological process and lower dosages of $\mathrm{Ky}$ - 
nurenine and IDO enzyme pre-transplant could identify patients at higher risk for developing aGVHD.

In this perspective, the initiation of the study of the metabolomics can effectively contribute to a better understanding of the mechanisms not yet identified in the pathophysiology of aGVHD. Future clinical studies including larger population may contribute to advance Knowledge in this area [21-29].

\section{Compliance with Ethical Standards}

\section{Conflict of interest}

All authors declare that they have no conflicts of interest related to this manuscript.

\section{Ethical appoval}

All procedures performed in studies involving human participants were in accordance with ethical standards of the institutional and/or national research comitte and with the 1964 Helsinki declaration and its later amendments or comparable ethical standards.

\section{Funding}

The study was supported by CNPq (Conselho Nacional de Desenvolvimento Científico e Tecnológico, Ministry of Education, Brazil).

\section{References}

1. Choi SW, Levine JE, Ferrara JL (2010) Pathogenesis and management of graft-versus-host disease. Immunol Allergy Clin North Am 30: 75-101.

2. Gratwohl A (2012) The EBMT risk score. Bone Marrow Transplant 47: 749-756.

3. Reikvam H, Hatfield K, Bruserud $\varnothing$ (2016) The pretransplant systemic metabolic profile reflects a risk of acute graft versus host disease after allogeneic stem cell transplantation. Metabolomics 12: 12.

4. Paczesny S, Raiker N, Brooks S, Mumaw C (2013) Graftversus-host disease biomarkers: Omics and personalized medicine. Int J Hematol 98: 275-292.

5. Ahmed SO, Ghavamzadeh A, Zaidi SZ, Baldomero H, Pasquini MC, et al. (2011 Trends of hematopoietic stem cell transplantation on the eastern mediterranean region. Biol Blood Marrow Transplant 17: 1352-1361.

6. Fuchs EJ (2012) Haploidentical transplantation for hematologic malignancies: Where do we stand? Hematology Am Soc Hematol Educ Program 2012: 230-236.

7. Korn T, Bettelli E, Oukka M, Kuchroo VK (2009) IL-17 and Th17 Cells. Annu Rev Immunol 27: 485-517.

8. Mándi $Y$, Vécsei $L$ (2012) The kynurenine system and immunoregulation. J Neural Transm (Vienna) 119: 197-209.

9. Janette Furuzawa-Carballeda, Guadalupe Lima, Perla Simancas, Dolores Ramos-Bello, Margaret Simancas, et al. (2012) Peripheral regulatory cells immunopheno typing in kidney transplant recipients with different clinical profiles: A cross-sectional study. J Transplant 2012: 256960.

10. Munn DH, Zhou M, Attwood JT, Bondarev I, Conway SJ, et al. (1998) Prevention of allogeneic fetal rejection by tryptophan catabolism. Science 281: 1191-1203.
11. Kamimura S, Eguchi K, Yonezawa M, Sekiba K (1991) Localization and developmental change of indoleamine 2,3-dioxygenase activity in the human placenta. Acta Med Okayama 45: 135-139.

12. Schröcksnadel H, Baier-Bitterlich G, Dapunt O, Wachter H, Fuchs D (1996) Decreased plasma tryptophan in pregnancy. Obstet Gynecol 88: 47-50.

13. Terness $P$, Bauer TM, Röse L, Dufter C, Watzlik A, et al. (2002) Inhibition of allogeneic T cell proliferation by indoleamine 2,3-dioxygenase-expressing dendritic cells: Mediation of suppression by tryptophan metabolites. J Exp Med 196: 447-457.

14. Dixit K, Khan MA, Sharma YD, Moinuddin, Alam K (2011) Peroxynitrite-induced modification of $\mathrm{H} 2 \mathrm{~A}$ histone presents epitopes which are strongly bound by human anti-DNA autoantibodies: Role of peroxynitrite-modified-H2A in SLE induction and progression. Hum Immunol 72: 219-225.

15. Duncan MW (2003) A review of approaches to the analysis of 3-nitrotyrosine. Amino Acids 25: 351-361.

16. Gonzalez-Gay MA, Llorca J, Sanchez E, Lopez-Nevot MA, Amoli MM, et al. (2004) Inducible but not endotelial nitric oxide synthase polymorphism is associated with susceptibility to rheumatoid arthritis in northwest spain. Rheumatology 43: $1182-1185$.

17. Ahsan H (2013) 3-Nitrotyrosine: A biomarker of nitrogen free radical species modified proteins in systemic auto immunogenic conditions. Hum Immunol 74: 1392-1399.

18. Ischiropoulos H (1998) Biological tyrosine nitration: A pathophysiological function of nitric oxide and reactive oxygen species. Arch Biochem Biophys 356: 1-11.

19. Khan F, Ali R (2006) Antibodies against nitric oxide damaged poly L-tyrosine and 3- nitrotyrosine levels in systemic lupus erythematosus. J Biochem Mol Biol 39: 189-196.

20. Khan F, Siddiqui AA (2006) Prevalence of anti-3-nitrotyrosine antibodies in the joint synovial fluid of patients with rheumatoid arthritis, osteoarthritis and systemic lupus erythematosus. Clin Chim Acta 370: 100-107.

21. Oates JC, Christensen EF, Reilly CM, Self SE, Gilkeson GS (1999) Prospective measure of serum 3-nitrotyrosine levels in systemic lupus erythematosus: Correlation with disease activity. Proc Assoc Am Physicians 111: 611-621.

22. Ohmori H, Kanayama N (2005) Immunogenicity of an inflammation associated product, tyrosine nitrated self-proteins. Autoimmun Rev 4: 224-229.

23. Ohmori $H$, Oka M, Nishikawa $Y$, Shigemitsu $H$, Takeuchi $M$, et al. (2005) Immunogenicity of autologous IgG bearing the inflammation-associated marker 3-nitrotyrosine. Immunol Lett 96: 47-54

24. Thomson L, Christie J, Vadseth C, Lanken PN, Fu X, et al. (2007) Identification of immunoglobulins that recognize 3-nitrotyrosine in patients with acute lung injury after major trauma. Am J Respir Cell Mol Biol 36: 152-157.

25. Schroten H, Spors B, Hucke C, Stins M, Kim KS, et al. (2001) Potential role of human brain microvascular endothelial cells in the pathogenesis of brain abscess: Inhibition of Staphylococcus aureus by activation of indoleamine 2,3-dioxygenase. Neuropedriatrics 32: 206-210.

26. Adams O, Besken K, Oberdorfer C, MacKenzie CR, Takikawa O, et al. (2004) Role of indoleamine 2,3-dioxygenase in alpha/beta and gamma interferon-mediated antiviral effects against herpes simplex virus infections. J Virology 78 : 2632-2636 
27. Mellor AL, Munn DH (1999) Tryptophan catabolism and T cell tolerance: Immunosuppression by starvation? Immunol Today 20: 469-473.

28. Grohmann U, Fallarino F, Puccetti P (2003) Tolerance, DCs and tryptophan: Much ado about IDO. Trends Immunol 24: 242-248.
29. Frumento G, Rotondo R, Tonetti M, Damonte G, Benatti U, et al. (2002) Tryptophan-derived catabolites are responsible for inhibition of $T$ and natural killer cell proliferation induced by indoleamine 2,3- dioxygenase. J Exp Med 196: 459-468. 
Annex 1: Duplicate metabolites evaluated from each plasma sample Kit Absolutei DQ p 180.

\section{Acylcarnitines}

\begin{tabular}{|c|c|c|c|}
\hline $\mathrm{CO}$ & Carnitine & C10: 1 & Decenocarnitine \\
\hline C2 & Acetylcarnitine & C10: 2 & Decadenylcarnitine \\
\hline C3 & Propionylcarnitine & C12 & Dodecanoylcarnitine \\
\hline C3: 1 & Propenoylcarnitine & C12: 1 & Dodecenoylcarnitine \\
\hline $\mathrm{C} 3-\mathrm{OH}$ & Hidroxypropionylcarnitine & C12-DC & Dodecanedioylcarnitine \\
\hline C4 & Butyrilcarnitine & C14 & Tetradecanoylcarnitine \\
\hline C4: 1 & Butenylcarnitine & C14: 1 & Tetradecanoylcarnitine \\
\hline C4-OH (C3-DC) & Idroxybutyrylcarnitine & C14: $1-\mathrm{OH}$ & Hydroxytetradecenoylcarnitine \\
\hline C5 & Valerylcarnitine & C14: 2 & Tetradecadienylcarnitine \\
\hline C5: 1 & Tiglilcarnitine & C14: 2-OH & Hydroxytetradecadienylcarnitine \\
\hline C5: 1-DC & Glutaconylcarnitine & C16 & Hexadecanoylcarnitine \\
\hline C5-DC (C6-OH) & $\begin{array}{l}\text { Glutarylcarnitine } \\
\text { (Hydroxyhexanoylcarnitine) }\end{array}$ & C16: 1 & Hexadecenoylcarnitine \\
\hline C5-M-DC & Methylglutarylcarnitine & C16: 1-OH & Hydroxyhexadecenoylcarnitine \\
\hline C5-OH (C3-DC-M) & Hydroxyvalerylcarnitine & C16: 2 & Hexadecadienylcarnitine \\
\hline C6 (C4: 1-DC) & $\begin{array}{l}\text { Hexanoylcarnitine } \\
\text { (Fumarylcarnitine) }\end{array}$ & C16: 2-OH & Hydroxyhexadecadienylcarnitine \\
\hline C6: 1 & Hexenoylcarnitine & $\mathrm{C} 16-\mathrm{OH}$ & Hydroxyhexadecanoylcarnitine \\
\hline C7-DC & Pyelinylcarnitine & C18 & Octadecanoylcarnitine \\
\hline C8 & Octanoylcarnitine & C18: 1 & Octadecenoylcarnitine \\
\hline $\mathrm{C9}$ & Nonailcarnitine & C18: 1-OH & Hydroxyoctadecenoylcarnitine \\
\hline C10 & Decanoylcarnitine & C18: 2 & Octadecadienylcarnitine \\
\hline
\end{tabular}

Amino Acids and Biogenic Amines

\begin{tabular}{|c|c|c|c|}
\hline Ala & Alanine & Ac-Orn & Acetylornithine \\
\hline Arg & Arginine & ADAM & Asymmetric Dimethylarginine \\
\hline Asn & Asparagine & SDMA & Symmetrical Dimethylarginine \\
\hline Asp & Aspartate & Alpha-AAA & Alpha Aminoapidic Acid \\
\hline Cit & Citrulline & Carnosine & Carnosine \\
\hline GIn & Glutamine & Creatine & Creatine \\
\hline Glu & Glutamate & Histamine & Histamine \\
\hline Gli & Glycine & Kyinurenine & Kynurenine \\
\hline His & Histidine & Met-SO & Methyoninesulfoxide \\
\hline Ile & Isoleucine & Nitro-tir & Nitrotyrosine \\
\hline Leu & Leucine & cis-OH-Pro & cis-4-hydroxyproline \\
\hline Lis & Lisine & trans-OH-Pro & trans-4-hydroxyproline \\
\hline Met & Methionine & PEA & Penylethylamine \\
\hline Orn & Ornithine & Putrescina & Putrescina \\
\hline Pen & Penilalanine & Sarcosine & Sarcosine \\
\hline Pro & Proline & Serotonin & Serotonin \\
\hline Ser & Serine & Spermidine & Spermidine \\
\hline Tre & Threonine & Spermina & Spermina \\
\hline Trp & Tryptophan & Taurine & Taurine \\
\hline Tir & Tyrosine & Dopamine & Dopamine \\
\hline Val & Valine & DOPA & DOPA \\
\hline
\end{tabular}




\section{Monosacharides}

Sum of Hexoses (Including glucose)

Sphingolipids

\begin{tabular}{|l|l|l|l|}
\hline SM (OH) C14: 0 & SM C18: 0 & SM (OH) C22: 1 & SM (OH) C24: 1 \\
\hline SM C16: 0 & SM C18: 1 & SM (OH) C22: 2 & SM C26: 0 \\
\hline SM C16: 1 & SM C20: 2 & SM C24: & SM C26: 1 \\
\hline SM (OH) C16: 1 & SM C22: 3 & SM C24: 1 & \\
\hline
\end{tabular}

\section{Glycerophospholipids}

\begin{tabular}{|c|c|c|c|}
\hline LisoPC a C14: 0 & PC aa C34: 1 & PC aa C42: 0 & PC ae C38: 2 \\
\hline LisoPC a C16: 0 & PC aa C34: 2 & PC aa C42: 1 & PC ae C38: 3 \\
\hline LisoPC a C16: 1 & PC aa C34: 3 & PC aa C42: 2 & PC ae C38: 4 \\
\hline LisoPC a C17: 0 & PC aa C34: 4 & PC aa C42: 4 & PC ae C38: 5 \\
\hline LisoPC a C18: 0 & PC aa C36: 0 & PC aa C42: 5 & PC ae C38: 6 \\
\hline LisoPC a C18: 1 & PC aa C36: 1 & PC aa C42: 6 & PC ae C40: 0 \\
\hline LisoPC a C18: 2 & PC aa C36: 2 & PC ae C30: 0 & PC ae C40: 1 \\
\hline LisoPC a C20: 3 & PC aa C36: 3 & PC ae C30: 1 & PC ae C40: 2 \\
\hline LisoPC a C20: 4 & PC aa C36: 4 & PC ae C30: 2 & PC ae C40: 3 \\
\hline LisoPC a C24: 0 & PC aa C36: 5 & PC ae C32: 1 & PC ae C40: 4 \\
\hline LisoPC a C26: 0 & PC aa C36: 6 & PC ae C32: 2 & PC ae C40: 5 \\
\hline LisoPC a C26: 1 & PC aa C38: 0 & PC ae C34: 0 & PC ae C40: 6 \\
\hline LisoPC a C28: 0 & PC aa C38: 1 & PC ae C34: 1 & PC ae C42: 0 \\
\hline LisoPC a C28: 1 & PC aa C38: 3 & PC ae C34: 2 & PC ae C42: 2 \\
\hline PC aa C24: 0 & PC aa C38: 4 & PC ae C34: 3 & PC ae C42: 3 \\
\hline PC aa C26: 0 & PC aa C38: 5 & PC ae C36: 0 & PC ae C42: 4 \\
\hline PC aa C28: 1 & PC aa C38: 6 & PC ae C36: 1 & PC ae C42: 5 \\
\hline PC aa C30: 0 & PC aa C40: 1 & PC ae C36: 2 & PC ae C44: 3 \\
\hline PC aa C30: 2 & PC aa C40: 2 & PC ae C36: 3 & PC ae C44: 4 \\
\hline PC aa C32: 0 & PC aa C40: 3 & PC ae C36: 4 & PC ae C44: 5 \\
\hline PC aa C32: 1 & PC aa C40: 4 & PC ae C36: 5 & PC ae C44: 6 \\
\hline PC aa C32: 2 & PC aa C40: 5 & PC ae C38: 0 & \\
\hline PC aa C32: 3 & PC aa C40: 6 & PC ae C38: 1 & \\
\hline
\end{tabular}

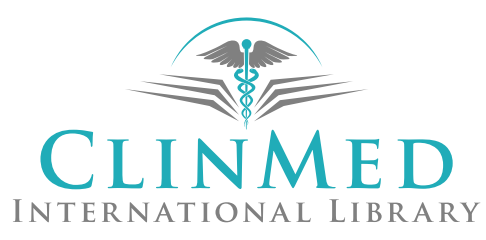

